\title{
Immune-mediated cochleovestibular disease
}

\author{
Suchan M, Kaliarik L, Krempaska S, Koval J \\ Department of Otorhinolaryngology and Head and Neck Surgery, L Pasteur University Hospital, Medical Faculty, \\ PJ Safarik University, Kosice, Slovakia. martinsuchan@zoznam.sk
}

\begin{abstract}
OBJECTIVE: The aim of this study is to prove the involvement of the immune response in the etiopathogenesis of some cochleovestibular disorders by a demonstration of antibodies against inner ear antigens and identify the benefits of immunosuppressive therapy.

BACKGROUND: McCabe in 1979 postulated the hypothesis of autoimmune inner ear disease.

METHODS: Sodium dodecyl sulfate polyacrylamid gel electrophoresis and immunoblotting were used to examine the serum of 74 subjects for the presence of antibodies against inner ear antigens. The subjects were divided into three groups: A - subjects with idiopathic progressive sensorineural hearing loss, B - subjects with Menière's disease, $\mathrm{C}$ - healthy subjects. Individuals with proven antibodies received immunosuppressive therapy. RESULTS: We detected antibodies against inner ear antigens with molecular weight of 30, 50, 60, 80, $100 \mathrm{kDa}$. In group A they were found in $52 \%$ of 25 subjects, in group B in $44 \%$ of 25 subjects and they were not detected in group C. An improvement of hearing was recorded in $69 \%$ of subjects in group A. An improvement of hearing was observed in $72 \%$, significant relief of vertigo in $81 \%$ of subjects in group B.

CONCLUSION: The present study supports the hypothesis of immune-mediated cochleovestibular disease (Tab. 3, Ref. 15). Text in PDF www.elis.sk.

KEY WORDS: immune-mediated cochleovestibular disease, immunosuppressive therapy.
\end{abstract}

\section{Introduction}

In clinical practice we often meet with cases of cochleovestibular disorders of unknown etiology. Acute, rapidly progressive sensorineural hearing loss is a special unit. McCabe (1) in 1979 postulated the hypothesis that bilateral, rapidly progressive, often fluctuating, sensorineural hearing loss with or without vestibular symptoms is a new clinical entity that is of autoimmune origin and termed it the autoimmune inner ear disease - AIED. An immunological process can damage both cochlear and vestibular function of the inner ear. It follows that some of Menière's disease cases may be a result of the immune response aimed against the inner ear. In the literature we can meet with a number of studies in the field of oto-immunology. Their aim is to prove the involvement of mechanisms of cellular and humoral immunity in damage to the auditory and vestibular function of the inner ear. Diseases of the inner ear with evidence of immune response, whether primary or secondary, localized or generalized, may be defined as conditionally immune-mediated cochleovestibular diseases.

The diagnosis of immune-mediated cochleovestibular disease is determined on the basis of clinical manifestations, laboratory

Department of Otorhinolaryngology and Head and Neck Surgery, L Pasteur University Hospital, Medical Faculty, PJ Safarik University, Kosice, Slovakia

Address for correspondence: M. Šuchaň, MD, Department of Otorhinolaryngology and Head and Neck Surgery, L. Pasteur University Hospital, Medical Faculty, P. J. Safarik University, Trieda SNP 1, SK-040 01 Kosice, Slovakia.

Phone: +421.55.6403735 evidence of an immune response directed against the inner ear and a positive response to immunosuppressive therapy.

The aim of our study was to demonstrate antibodies against inner ear antigens in the serum of subjects with rapidly progressive sensorineural hearing loss and Menière's disease, to confirm the role of humoral immune mechanisms in their etiology and discover the benefits of immunosuppressive therapy in a group of subjects with detected antibodies against antigens of the inner ear.

\section{Materials and methods}

Subjects with unilateral and bilateral progressive sensorineural hearing loss of unknown etiology and subjects with Menière's disease were enrolled in the prospective study that we conducted at the Department of Otorhinolaryngology and Head and Neck Surgery, L. Pasteur University Hospital and Medical Faculty, P. J. Safarik University, Kosice, Slovakia in the period from 2010 to 2013. In the study, we examined the sera of 74 subjects for the presence of antibodies against antigens of the inner ear. Subjects were divided into three groups. Group A -25 subjects with unilateral or bilateral progressive sensorineural hearing loss of unknown etiology. Group B - 25 subjects with Menière's disease diagnosed by AAO-HNS 1995 (2) and positive result of electrocochleography (ECochG) test. Severity of Menière's disease was determined by clinical status, ECochG, electronystagmography (ENG) and vestibular evoked myogenic potentials (VEMPs) (3). Group C -24 healthy individuals without disorder of the inner ear and no personal or family history of autoimmune disease. 
Group A - 25 subjects, 13 women and 12 men. The mean age was 40.2 years (23-64 years). Unilateral progressive sensorineural hearing loss was observed in 8 subjects, bilateral in 18 subjects.

Group B - 25 subjects, 17 women and 8 men. The mean age was 44.5 years (18-64 years). Unilateral Menière's disease was found in 22 subjects, bilateral in 3 subjects.

Group C -24 healthy subjects, 12 women and 12 men, who formed the control group. The mean age was 33 years (18-60 years).

Subjects underwent a battery of examinations (history, otomicroscopic examination, pure tone threshold audiometry, speech audiometry, tympanometry, stapedius reflex examination, OAE, BERA, VEMP, ENG, ECochG, examination by imaging techniques - CT of temporal bones, MRI of brain and PC angles, laboratory tests), in order to rule out other causes of the cochleovestibular disease, such as presbyacusis (age-related hearing loss), auditory trauma, ototoxicity, infection, vascular disorder, cancer, hereditary hearing loss, and others.

We performed the following laboratory tests: blood count, differential blood count, sedimentation of red blood cells (ESR), screening for syphilis (TPH, RPR), quantitative examination of IgA, IgG, IgM, complement examination, cryoglobulins, CIK, examination of antinuclear antibodies (ANA), antibodies against extracted nuclear antigen (ENA) and antibodies against cytoplasm of neutrophils (ANCA).

The method of sodium dodecyl sulfate polyacrylamide gel electrophoresis (SDS-PAGE) and immunoblotting (4) was used to attempt to demonstrate specific antibodies against inner ear antigens in the serum of investigated subjects.

Subjects with evidence of antibodies against inner ear antigens received immunosuppressive therapy - prednisone $1 \mathrm{mg} / \mathrm{kg} / \mathrm{d}$ at the maximum dose of $60 \mathrm{mg}$ / day for 4 weeks followed by a taper to the lowest possible dose required to treat hearing symptoms. If there was no response after 4 weeks high-dose prednisone, prednisone was rapidly tapered and no further additional immunosuppressive regimen was recommended.

Average improvement in pure tone threshold by at least $10 \mathrm{~dB}$ at the frequency of 500, 1000 and $2000 \mathrm{~Hz}$ and/or a $12 \%$ improvement in speech discrimination score were considered a positive response to treatment. The effect of immunosuppressive therapy on vertigo in subjects with Menière's disease was evaluated according to the AAO-HNS, 1995 criteria for the evaluation of treatment of subjects with Menière's disease (2).

\section{Results}

\section{Group A}

In the group of 25 subjects with rapidly progressive sensorineural hearing loss, SDS-PAGE and immunoblotting was used to confirm antibodies against inner ear antigens in $13(52 \%)$ of them. In case of unilateral progressive sensorineural hearing loss - in one $(1 / 7-14 \%)$ subject, in bilateral hearing loss - in $12(12 / 18$ $-66.6 \%$ ) subjects.

The antibodies against antigens of the inner ear have been found in approximately equal numbers in women (54\%) and in men $(50 \%)$.
Tab. 1. Progressive sensorineural hearing loss - results of laboratory tests and therapy.

\begin{tabular}{|c|c|c|c|c|c|c|}
\hline No & $\mathrm{Dg}$ & Age & Gender & $\begin{array}{c}\text { Non- } \\
\text { specific } \\
\text { antibodies }\end{array}$ & $\begin{array}{l}\text { Inner ear } \\
\text { antibodies }\end{array}$ & $\begin{array}{l}\text { Result of } \\
\text { therapy }\end{array}$ \\
\hline 1 & BSNHL & 43 & $\mathrm{~F}$ & ENA & $30,60 \mathrm{kDa}$ & - \\
\hline 2 & BSNHL & 26 & M & - & $50 \mathrm{kDa}$ & + \\
\hline 3 & BSNHL & 23 & M & - & $60 \mathrm{kDa}$ & + \\
\hline 4 & BSNHL & 37 & $\mathrm{~F}$ & - & $30,50,80 \mathrm{kDa}$ & + \\
\hline 5 & BSNHL & 29 & $\mathrm{~F}$ & ANA & $30,60 \mathrm{kDa}$ & + \\
\hline 6 & BSNHL & 29 & M & - & $100 \mathrm{kDa}$ & Stabilisation \\
\hline 7 & BSNHL & 64 & M & - & - & \\
\hline 8 & BSNHL & 35 & $\mathrm{~F}$ & - & - & \\
\hline 9 & BSNHL & 58 & M & - & - & \\
\hline 10 & BSNHL & 35 & M & - & - & \\
\hline 11 & USNHL & 44 & M & - & $60 \mathrm{kDa}$ & + \\
\hline 12 & USNHL & 45 & $\mathrm{~F}$ & - & - & \\
\hline 13 & USNHL & 27 & $\mathrm{~F}$ & - & - & \\
\hline 14 & USNHL & 33 & $\mathrm{~F}$ & - & - & \\
\hline 15 & BSNHL & 45 & $\mathrm{~F}$ & - & $50,100 \mathrm{kDa}$ & - \\
\hline 16 & BSNHL & 36 & M & - & $50 \mathrm{kDa}$ & + \\
\hline 17 & BSNHL & 43 & $\mathrm{~F}$ & - & $30,60 \mathrm{kDa}$ & + \\
\hline 18 & USNHL & 54 & M & - & - & \\
\hline 19 & BSNHL & 61 & $\mathrm{~F}$ & - & $50 \mathrm{kDa}$ & Stabilisation \\
\hline 20 & USNHL & 28 & $\mathrm{~F}$ & - & - & \\
\hline 21 & BSNHL & 36 & M & - & $100 \mathrm{kDa}$ & + \\
\hline 22 & BSNHL & 33 & $\mathrm{~F}$ & - & $30,60 \mathrm{kDa}$ & + \\
\hline 23 & BSNHL & 55 & $\mathrm{~F}$ & - & - & \\
\hline 24 & USNHL & 47 & M & - & - & \\
\hline 25 & BSNHL & 39 & M & - & - & \\
\hline
\end{tabular}

BSNHL - bilateral sensorineural hearing loss, USNHL - unilateral sensorineural hearing loss, $\mathrm{F}$ - female, $\mathrm{M}$ - male, + positive, - negative

The presence of antibodies against antigens of the inner ear was noted particularly in subjects in the third (6), 4th (8) and 5th(6) decade. The mean age of the subjects, in whom antibodies against antigens of the inner ear were demonstrated, was 37 years, median 36 years.

SDS PAGE and immunoblotting were used to detect antibodies against inner ear antigens of molecular weight 30, 50, 60, 80 and $100 \mathrm{kDa}$ in the sera of the examined subjects.

In two $(15 \%)$ study subjects with specific antibodies against inner ear antigens we also found non-specific antibodies ANA and ENA.

Consequently, subjects with confirmed antibodies against inner ear antigens underwent immunosuppressive therapy with corticosteroids. .During the reporting period, improvement of hearing was observed in 7 (54\%), stabilization of hearing loss in $2(15 \%)$ subjects. Treatment was not successful in four (31\%) subjects. Results of laboratory tests and therapy are documented in Table 1.

\section{Group B}

In the group of 25 subjects with Menière's disease, SDS PAGE and immunoblotting confirmed antibodies against inner ear antigens in $11(44 \%)$ of them. In subjects with unilateral Menière's disease - in $9(22.9-41 \%)$, in subjects with bilateral Menière's disease - in $2(2 / 3-66 \%)$.

The antibodies against antigens of the inner ear were found more often in women $(47 \%)$ than in men $(37.5 \%)$.

The presence of antibodies against antigens of the inner ear was observed mainly in elderly subjects ( 5 th to 7 th decade) -73 
Tab. 2. Menière's disease - results of laboratory tests and therapy.

\begin{tabular}{cccccccc}
\hline No. & Dg. & Class & Age & Gender & $\begin{array}{c}\text { Non- } \\
\text { specific } \\
\text { antibodies }\end{array}$ & $\begin{array}{c}\text { Inner ear } \\
\text { antibodies }\end{array}$ & $\begin{array}{c}\text { Result of } \\
\text { therapy }\end{array}$ \\
\hline 1 & U & A & 18 & F & - & - & \\
2 & U & B & 21 & F & - & $30,50,80 \mathrm{kDa}$ & + , class A \\
3 & U & B & 32 & M & - & - & \\
4 & U & B & 52 & F & - & $30,60 \mathrm{kDa}$ & Stab., class B \\
5 & U & C & 55 & F & - & - & \\
6 & U & B & 51 & F & - & $30,60 \mathrm{kDa}$ & - , class B \\
7 & B & D & 44 & F & ANA & $30,50,80 \mathrm{kDA}$ & - , class D \\
8 & U & B & 58 & M & - & - & \\
9 & U & B & 41 & M & - & - & \\
10 & U & B & 64 & M & - & $50 \mathrm{kDa}$ & + , class B \\
11 & U & C & 37 & F & - & - & \\
12 & U & B & 57 & M & - & $50,100 \mathrm{kDa}$ & + , class A \\
13 & U & B & 43 & F & - & - & \\
14 & U & B & 57 & F & - & - & \\
15 & U & C & 53 & F & - & $50,100 \mathrm{kDa}$ & Stab., class B \\
16 & U & B & 56 & F & - & - & \\
17 & B & C & 38 & M & - & $30,60 \mathrm{kDa}$ &,- class D \\
18 & B & B & 33 & F & ANA & - & \\
19 & U & B & 49 & F & - & - & \\
20 & U & B & 32 & F & - & $60 \mathrm{kDa}$ & Stab., class B \\
21 & U & C & 46 & F & ENA & $100 \mathrm{kDa}$ &,+ class B \\
22 & U & D & 43 & M & - & - & \\
23 & U & C & 53 & F & - & - & \\
24 & U & B & 51 & F & - & $60 \mathrm{kDa}$ & + , class A \\
25 & U & A & 28 & M & - & - & \\
\hline
\end{tabular}

B - bilateral Menière's disease, U - unilateral Menière's disease, Stab. - stabilisation, + positive, - negative

Tab. 3. Frequency of antibodies against inner ear antigens.

\begin{tabular}{lccccc}
\hline IEA & $30 \mathrm{kDa}$ & $50 \mathrm{kDa}$ & $60 \mathrm{kDa}$ & $80 \mathrm{kDa}$ & $100 \mathrm{kDa}$ \\
\hline $\mathrm{SNHL}$ & 5 & 5 & 6 & 1 & 2 \\
MD & 4 & 5 & 5 & 2 & 3 \\
\hline
\end{tabular}

IEA - inner ear antigens, SNHL - sensorineural hearing loss, MD - Menière's disease

$\%$. The mean age of the subjects, with demonstrated antibodies against antigens of the inner ear, was 44.5 years, median 51 years.

SDS PAGE and immunoblotting were used to investigate the sera of subjects to detect antibodies against inner ear antigens of molecular weight 30, 50, 60, 80 and $100 \mathrm{kDa}$.

In two (18\%) study subjects with specific antibodies against inner ear antigens we also found non-specific antibodies ANA and ENA.

Consequently, subjects with confirmed antibodies against inner ear antigens underwent immunosuppressive therapy with corticosteroids. During the reporting period we observed improvement of hearing in $5(45 \%)$, stabilization of hearing loss in $3(27 \%)$ subjects. Reduction in the frequency and intensity of vertigo was reported in $9(81 \%)$ subjects. According to the criteria of AAOHNS, 1995 (2) for the success of the treatment of Menière's disease, immunosuppressive therapy allowed complete control of vertigo attacks in 3 subjects (class A), significant reduction in 6 subjects (class B) and only partial reduction in 2 (class D). Results of laboratory tests and therapy are documented in Table 2.

The frequency of detection of antibodies against inner ear antigens in subjects with unilateral or bilateral idiopathic progressive sensorineural hearing loss and Menière's disease is documented in Table 3.
Group C

In the control group no antibodies against inner ear antigens were detected. Non-specific antibodies were not detected as well.

\section{Discussion}

The autoimmune inner ear disease with characteristic clinical picture (rapidly progressive, often fluctuating sensorineural hearing loss with / without vestibular symptomatology with good response to immunosuppressive therapy) cannot be considered without evidence of an immune response. In the literature, we observe the effort to develop tests that would confirm the involvement of mechanisms of humoral and cellular immunity in the etiopathogenesis of cochleovestibular disorders. They are based on the demonstration of antibodies or activated $T$ cells against antigens of the inner ear in the serum of subjects. Using the method of Western blot analysis and two-dimensional electrophoresis Harris (5) was able to demonstrate antibodies against antigens of the inner ear with a molecular weight of $68 \mathrm{kDa}$. The same method was used by Boulassel (6) to find in the serum of $44 \%$ of subjects the antibodies against antigens with molecular weight of 30, 42 and $68 \mathrm{kDa}$. Lejeune and Charachone (7) used an immunoperoxidase test to demonstrate the presence of antibodies against antigens of molecular weight 130, 45, 22 and $14 \mathrm{kDa}$. Kosaka (8) used Western blot analysis to detect antibodies against antigens of molecular weight $33-35 \mathrm{kDa}, 42 \mathrm{kDa}$ and $68 \mathrm{kDa}$. Alleman (9) used immunohistochemistry and fluorescent microscopy to demonstrate immunoglobulin $\mathrm{G}(\mathrm{IgG})$ bound to saccus endolymphaticus in $10 \%$ of subjects with Menière's disease. Similarly, Soliman (10) used indirect immunofluorescence to detect the presence of immunoglobulins in the epithelium of endolymphatic sac and perisacular stroma in up to $40 \%$ of the examined subjects with Menière's disease. Yoo (11) proved antibodies against collagen type II in the sera of subjects with Menière's disease and otosclerosis. They were found in endolymphatic duct, spiral ligament in enchondral zone in otic capsule, which highlights the importance of type II collagen in the pathogenesis of these disorders. In our study we used SDS PAGE and immunoblotting to demonstrate in the serum of subjects with progressive sensorineural hearing loss (group A), and Menière's disease (group B) antibodies against inner ear antigens of molecular weight $30,50,60,80$ and $100 \mathrm{kDa}$. The antibodies were dominated in the groups $\mathrm{A}$ and $\mathrm{B}$ by antibodies against inner ear antigens of molecular weight 60,50 and $30 \mathrm{kDa}$ (Tab. 3). In the control group, none of the subjects were shown to have antibodies against antigens of the inner ear. The presented overview demonstrates high variability of the results. Antibodies against inner ear antigens were detected with different molecular weight, which is probably due to different methods used to collect and process the material. The issue is the sensitivity and specificity of individual tests. Essentially, however, the presence of antibodies against antigens of the inner ear proves the involvement of immune system in the damage to the inner ear, supporting the hypothesis of the existence of an immune-mediated disease of the inner ear.

Among subjects with progressive sensorineural hearing impairment, we found the presence of antibodies against antigens of the inner ear in $52 \%$ of examined subjects, and in the group 
with Menière's disease - in $44 \%$ of examined subjects. Our results are similar to those presented in works of Boulassel (6) and Soliman (10) mentioned above. In both groups in case of bilateral involvement of the inner ear, we confirmed antibodies against inner ear antigens in up to $2 / 3$ subjects. These results correlate with the characteristic features of AIED, typical for frequent bilateral involvement of the inner ear associated with sensorineural hearing loss and vestibular symptomatology.

In our study we found that the immune-mediated disorder with cochleovestibular disease with proven antibodies against antigens of the inner ear affects people in the middle and higher age, most often in a 4th to 6th decade of life. Longer interrelation of external (infection, trauma, allergy etc.) and internal (genetic) factors can be responsible for increased presentation of inner ear antigens with consequent creation of antibodies.

In case of suspected immune-mediated cochleovestibular disorder, it is necessary, in addition to the examination of antibodies against inner ear antigens, to perform a series of laboratory tests to disprove or prove systemic autoimmune disease. Impairment of the inner ear might be secondary to systemic autoimmune disease. In our population, next to the antibodies against antigens of the inner ear, we also confirmed the non-specific antibodies (ANA, ENA) in two (14\%) of the subjects with progressive sensorineural hearing loss, and in two (18\%) of the subjects with Menière's disease. Systemic autoimmune disease may coexist in up to $15-30 \%$ of subjects (12).

The presence of antibodies against inner ear antigens helps us identify a group of subjects in whom we can expect the success of immunosuppressive therapy. So far, there is no model of immunosuppressive therapy. General treatment recommendations include prednisone $60 \mathrm{mg} /$ day or $1 \mathrm{mg} / \mathrm{kg} /$ day for 2-4 weeks $(13,14,15)$. Broughton (15) in his study found that $70 \%$ patients were responsive to high-dose corticosteroids therapy. Systemic corticosteroids therapy was used in the group A (progressive sensorineural hearing loss) to achieve improvement in hearing (7/13) in $54 \%$, stabilization in $(2 / 13) 15 \%$ of treated subjects. In the group B (Menière's disease) we observed improvement in hearing in (5/11) $45 \%$, stabilization in (3/11) $27 \%$, complete control of vertigo (class A) or significant limitation (class B) in (9/11) $81 \%$ of subjects. A positive response to this treatment indirectly demonstrates the role of immunity and supports the theory of immune-mediated cochleovestibular disorders. Clinical improvement in immune-mediated cochleovestibular disorders may be only temporary. In the long run, the effect of immunosuppressive therapy may significantly decrease (from $70 \%$ to $14 \%$ ) as reported by Broughton (15). In case of treatment failure, cytotoxic agents such as methotrexate, cyclophosphamide, azathioprine can be used, generally with limited modest response $(14,15)$. Chronic administration of toxic immunosuppressive therapy to improve or stabilize the hearing is associated with many serious side-effects. In the case of treatment failure and severe sensorineural hearing loss we prefer the correction of hearing using cochlear implants.

\section{Conclusion}

SDS PAGE and immunoblotting were used to successfully confirm the specific antibodies against the antigens of the inner ear with a molecular weight of $30,50,60,80$ and $100 \mathrm{kDa}$, and thus prove the involvement of the humoral immune response in the etiopathogenesis of some cochleovestibular disorders.

The presence of specific antibodies against antigens of the inner ear helps to identify a group of subjects in which we can assume the success of immunosuppressive therapy.The success of immunosuppressive therapy indirectly confirms the soundness of the hypothesis stipulating the existence of immune-mediated disease of the inner ear.

\section{References}

1. McCabe BF. Autoimmune sensorineural hearing loss. Ann Otol Rhinol Laryngol 1979; 88 (5 Pt 1): 585-589.

2. Committee of Hearing and Equilibrium. Guidelines for the Diagnosis and Evaluation of Therapy in Meniere's disesase. American Academy of Otolaryngology-Head and Neck Foundation, Inc. Otolaryngol Head Neck Surg 1995; 133 (3): 181-185.

3. Krempaska S, Koval J. The role of vestibular evoked myogenic potentials (VEMPs) in vestibulopahty diagnostics. Bratisl Lek Listy 2012; 113 (5): 301-306.

4. Rosocha J, Koval' J, Šuchaň M, Elbertová A, Krempaská S. Príspevok k objasneniu etiológie Meniérovej choroby. Otorinolaryngol. a Foniat. (Prague) 2001; (3): 172-177.

5. Harris JP, Sharp PA. Inner ear autoantibodies in patients with rapidly progressive sensorineural hearing loss. Laryngoscope 1990; 100 (5): $516-524$.

6. Boulassel MR, Deggouj N, Tomasi JP, Gersdorff M. Inner ear autoantibodies and their targets in patients with autoimmune inner ear diseases. Acta Otolaryngol 2001; 121 (1): 28-34.

7. Lejeune JM, Charachon R. New immunobiological tests in the investigation of Meniere's disease and sensorineural hearing loss. Acta Otolaryngol (Stockh) 1992; 112 (2): 174-179.

8. Kosaka K, Yamanobe S, Tomiyama S, Yagi T. Inner ear autoantibodies in patients with sensorineural hearing loss. Acta Otolaryngol Suppl 1995; (519): 176-177.

9. Alleman AM, Dornhoffer JL, Arenberg IK, Walker PD. Demonstration of autoantibodies to the endolymphatic sac in Meniere's disease. Laryngoscope 1997; 107 (2): 211-215.

10. Soliman AM. A subpopulation of Meniere's patients produce antibodies that bind to endolymphatic sac antigens. Am J Otol 1996; 17 (1): 76-80.

11. Yoo TJ, Stuart JM, Kang AH, Townes AS, Tomoda K, Dixit S. Type II colagen autoimmunity in Otosclerosis and Meniere's disease. Science 1982; 217 (4565): 1153-1154.

12. Bovo R, Aimoni C, Martini A. Immune-mediated inner ear disease. Acta Otolaryngol 2006; 126 (10): 1012-1021.

13. Rahman MU, Poe DS, Choi HK. Autoimmune vestibulo-cochlear disorders. Curr Opin Rheumatol 2001; 13 (3): 184-189.

14. Ruckenstein MJ. Autoimmune inner ear disease. Curr Opin Otolaryngol Head Neck Surg 2004; 12 (5): 426-430.

15. Broughton SS, Meyerhoff WE, Cohen SB. Immune-mediated inner ear disease: 10-year experience. Semin Arthritis Rheum 2004; 34 (2): 544-548.

Received May 11, 2015. Accepted September 15, 2015. 